\title{
Eficiência no uso de nitrogênio e correlação fenotípica em populações tropicais de milho no Tocantins ${ }^{1}$
}

\author{
Nitrogen use efficiency and phenotypic correlations of tropical maize populations in \\ Tocantins
}

\author{
Leandro Lopes Cancellier ${ }^{2 *}$, Flávio Sérgio Afférri ${ }^{3}$, Edmar Vinicius de Carvalho ${ }^{4}$, Michel Antônio Dotto ${ }^{4}$ e \\ Fernando Ferreira Leão ${ }^{5}$
}

\begin{abstract}
Resumo - O objetivo deste trabalho foi avaliar a eficiência no uso do nitrogênio e a correlação fenotípica de 24 populações de milho no Sul do Tocantins. Foram realizados dois experimentos, cada um correspondendo a um nível de adubação nitrogenada (alta e baixa) plantados no dia 21/11/2009. Os tratamentos foram constituídos de 24 populações obtidas do Programa de Melhoramento de Milho da Universidade Federal do Tocantins e uma variedade comercial, BR 106, usada como testemunha. O delineamento experimental utilizado, nos dois experimentos, foi blocos casualisados com duas repetições, sendo avaliado o peso de 100 sementes, peso hectolítrico, produtividade, e os índices de eficiência de uso do nitrogênio segundo metodologia proposta por Fischer et al. (1983) e Moll et al. (1982). Os genótipos 12-6; 25-5; 35-5; 12-2; 12-3 e 1-5 formaram o grupo das populações de maiores produtividades independente da condição de $\mathrm{N}$ no solo, com médias variando de 6.348 a $5.774 \mathrm{~kg} \mathrm{ha}^{-1}$. Pela metodologia de Moll foi possível diferenciar populações quanto à eficiência no uso de nitrogênio, no ambiente de baixa disponibilidade de $\mathrm{N}$ no solo. As características estudadas foram influenciadas pelos diferentes níveis de nitrogênio aplicados, exceto para a característica de peso de 100 sementes. As populações avaliadas apresentaram diferenças entre si, em todas as características estudadas, indicando variabilidade. O índice de Moll apresentou associação significativa com todas as características, exceto para o índice de Fischer, indicando ser possível a obtenção de genótipos eficientes no uso de nitrogênio por meio de seleção destas características.
\end{abstract}

Palavras-chave - Milho. Melhoramento genético. Plantas-efeito do nitrogênio.

\begin{abstract}
The objective of this study was to evaluate the nitrogen efficiency use and phenotypic correlation of 24 maize populations in Tocantins. Two experiments were conducted, each corresponding to a level of fertilization (high and low $\mathrm{N}$ ) planted on 21/11/2009, treatments consisted of 24 populations obtained from the Maize Breeding Program of Federal University of Tocantins and a commercial variety, BR 106, used as control. The experimental design in both experiments was randomized blocks with two replicates, and evaluated 100 seeds weight, hectoliter weight, yield and nitrogen use efficiency according to the methodology by Fischer et al. (1983) and Moll et al. (1982). The genotypes 12-6; 25-5; 35-5; 12-2; 12-3 and 1-5 formed the group of genotypes of higher yields, independent of soil $\mathrm{N}$, with averages ranging from 6,348 to $5,774 \mathrm{~kg} \mathrm{ha}^{-1}$. By the methodology of Moll was possible to differentiate populations for nitrogen use efficiency in environment of low availability of soil N. The characteristics evaluated were influenced by different levels of nitrogen, except for 100 seeds weight. The populations studied differ itself in all characteristics, indicating variability. Moll index was significantly associated with all characteristics studied except for the Fischer index, indicating possible to obtain genotypes efficient in nitrogen use by selection of these characteristics.
\end{abstract}

Key words - Maize. Genetic breeding. Plants-efects of nitrogen.

\footnotetext{
*Autor para correspondência

${ }^{1}$ Recebido para publicação em 03/06/2010; aprovado em 18/01/2011

Parte da dissertação de Mestrado do primeiro autor, apresentada ao Programa de Pós-Graduação em Produção Vegetal/UFT

${ }^{2}$ Programa de Pós-Graduação em Agronomia/Fitotecnia/UFLA, Lavras-MG, Brasil, 37.200-000, leandrocancellier@hotmail.com

${ }^{3}$ Laboratório de Sementes/UFT, Campus de Gurupi, Caixa Postal 66, Gurupi-TO, Brasil, 77.402-970, flavio@uft.edu.br

${ }^{4}$ Programa de Mestrado em Produção Vegetal/UFT, Gurupi-TO, Brasil, 77.410-000, ed.vinicius@hotmail.com, michelantoniodotto@hotmail.com

${ }^{5}$ Universidade Federal do Tocantins/UFT, Campus de Gurupi, Caixa Postal 66, Gurupi-TO, Brasil, 77.402-970, ferleao@hotmail.com
} 


\section{Introdução}

No Brasil, grande parte da produção de milho é realizada por pequenos e médios agricultores e com algum tipo de estresse ambiental. A seleção de cultivares próprios para ambientes pobres em nitrogênio $(\mathrm{N})$ tem sido buscada por diversos pesquisadores (MAJEROVICKS et al., 2002).

A produtividade do milho na região Norte é baixa por causa, principalmente, das altas temperaturas, do baixo nível tecnológico dos produtores e da insuficiência de sementes melhoradas de variedades adaptadas às condições de estresses abióticos (CARVALHO; SOUZA, 2007).

O milho é uma das culturas mais exigentes em fertilizantes, especialmente os nitrogenados. O suprimento inadequado de nitrogênio é considerado um dos principais fatores limitantes ao rendimento de grãos do milho, pois o $\mathrm{N}$ exerce importante função nos processos bioquímicos da planta. Ele é constituinte de proteínas, coenzimas, ácidos nucleicos, fitocromos, ATP, clorofila e inúmeras enzimas (JAKELAITIS et al., 2005), influenciando o crescimento da planta mais do que qualquer outro nutriente (BREDEMEIER; MUNDSTOCK, 2000).

O uso racional da adubação nitrogenada é fundamental, não somente para aumentar a eficiência de recuperação, mas também para aumentar a produtividade da cultura e diminuir o custo de produção (FAGERIA et al., 2007). Genótipo com alta eficiência no uso de $\mathrm{N}$ é desejável na agricultura de baixos insumos e também na agricultura capitalizada. Isto porque os desperdícios e a escassez desse elemento mineral, que é o mais exigido pelas plantas, podem gerar problemas econômicos, ambientais, de saúde pública e de segurança alimentar (ROESCH et al., 2005).

Dada a sua importância e a alta mobilidade no solo, o nitrogênio tem sido intensamente estudado, no sentido de maximizar a eficiência do seu uso, assim, temse procurado diminuir as perdas do nitrogênio no solo, bem como melhorar a absorção e a metabolização do N no interior da planta (BREDEMEIER; MUNDSTOCK, 2000). A seleção de genótipos com maior eficiência na utilização de nitrogênio é considerada, uma das maneiras mais adequadas para diminuir o custo de produção das culturas (MAJEROWICZ, et al., 2002).

$\mathrm{O}$ desenvolvimento de cultivares adaptadas às condições de estresse nitrogenado apresenta-se como uma opção economicamente viável e ecologicamente sustentável para garantir maior produtividade em sistemas agrícolas com baixa utilização de insumos (SOUZA et al., 2008).

Para Majerowicz et al. (2002) um caminho para aumentar a eficiência de uso do nitrogênio (EUN) é o melhoramento genético, que pode gerar milhos produtivos para solos pobres em N. Assim sugere-se o desenvolvimento de programas de melhoramento locais como a solução mais viável para o aumento da produção em sistemas agrícolas com baixa utilização de insumos.

Segundo Coimbra et al. (2010) as populações são menos produtivas que as cultivares modernas, embora apresentem uma complexa estrutura genética, sendo importantes fontes de variabilidade na busca por genes responsáveis pela tolerância ou resistência aos fatores bióticos e abióticos. Silva et al. (2008) afirma existir evidências experimentais que uma população de milho tropical foi eficiente em condições de estresse de $\mathrm{N}$, comprovando que populações de milho possuem variabilidade genética suficiente para que se tenha sucesso em um programa de melhoramento para condições de baixa disponibilidade de nitrogênio.

O objetivo deste trabalho foi avaliar a eficiência no uso do nitrogênio e a correlação fenotípica de 24 populações de milho no Sul do estado do Tocantins.

\section{Material e métodos}

Foram realizados dois experimentos, cada um correspondendo a um nível diferente de adubação nitrogenada em cobertura, sendo um experimento com baixo nível de $\mathrm{N}$, correspondendo a dose de $0 \mathrm{~kg} \mathrm{ha}^{-1} \mathrm{e}$ outro experimento com alto nível de $\mathrm{N}$, correspondendo a $150 \mathrm{~kg} \mathrm{ha}^{-1}$, sendo plantados em área experimental no município de Gurupi - TO, no dia 21 de novembro de 2009. Os tratamentos foram constituídos de 24 populações obtidas do Programa de Melhoramento de Milho da Universidade Federal do Tocantins e uma variedade comercial como testemunha (BR 106).

O delineamento experimental utilizado nos dois experimentos foi blocos casualisados com duas repetições. Para a instalação dos experimentos, foi utilizado o sistema de preparo de solo tipo convencional, com uma gradagem e após foram feitos sulcos com o espaçamento de $0,9 \mathrm{~m}$ entre linhas. As parcelas foram constituídas por duas linhas de três metros lineares cada.

O plantio das sementes e a adubação no sulco foram feitas manualmente. A adubação de plantio foi realizada utilizando $600 \mathrm{~kg} \mathrm{ha}^{-1}$ do adubo formulado 414-8, correspondendo às doses de NPK, respectivamente, proporcionando totais de 24 e $174 \mathrm{~kg} \mathrm{ha}^{-1}$, para os ambientes de baixo e alto $\mathrm{N}$, respectivamente. Plantou-se o milho na parcela a fim de obter 50 mil plantas ha $^{-1}$. A adubação de cobertura no experimento de alto $\mathrm{N}$ foi realizada dia 11/12/2009 utilizando-se como fonte a uréia. Os tratos culturais como o controle de plantas daninhas, pragas e doenças foram realizados de acordo com as recomendações técnicas da cultura. 
As seguintes características foram avaliadas: peso de 100 sementes - peso, em gramas, de amostras de 100 grãos, corrigido para $13 \%$ de umidade; pesos hectolítrico - peso, em $\mathrm{kg}$, de cem litros de grãos, corrigido para $13 \%$ de umidade e produtividade de grãos - que foi obtido pela pesagem dos grãos debulhados, em $\mathrm{kg}_{\text {parcela }}{ }^{-1}$, corrigidos para $13 \%$ de umidade e posteriormente convertidos os valores para $\mathrm{kg} \mathrm{ha}^{-1}$.

Foi avaliado a eficiência de uso de nitrogênio (EUN) segundo metodologia de Fischer et al. (1983) - índice utilizado para realizar seleções simultâneas em ambientes de baixo e alto nitrogênio, obtido pela Equação 1:

$$
\mathrm{EUN}=[\mathrm{Ya}(-\mathrm{N}) / \mathrm{Ya}(+\mathrm{N})] \mathrm{X}[\mathrm{Yx}(-\mathrm{N}) / \mathrm{Yx}(+\mathrm{N})]
$$

onde $\mathrm{Ya}(-\mathrm{N})$ é a produção do genótipo "a" sob baixo $\mathrm{N} ; \mathrm{Ya}(+\mathrm{N})$ é a produção do genótipo "a" sob alto $\mathrm{N}$; $\mathrm{Yx}(-\mathrm{N})$ é a produção média de todos os genótipos sob baixo $\mathrm{N}$; $\mathrm{Yx}(+\mathrm{N})$ é a produção média de todos os genótipos sob alto $\mathrm{N}$.

Também avaliou-se a eficiência de uso de nitrogênio segundo metodologia de Moll et al. (1982) obtida pela Equação 2:

$$
\mathrm{EUN}=\mathrm{Gw} / \mathrm{Ns}
$$

onde Gw é a massa de grãos e Ns é a massa de $\mathrm{N}$ aplicado no solo.

Obtidos os dados, foi realizada a análise de variância conjunta e aplicado o teste de agrupamento de Scott-Knott (1974) para as variáveis quando ocorreram diferenças significativas pelo teste F. Foi realizada também a correlação de Spearman entre as características e aplicado o teste t para verificar a significância da correlação.

\section{Resultados e discussões}

Na Tabela 1 pode-se observar que para as populações houve diferenças significativas $(p<0,01)$ para todas as características avaliadas com exceção apenas da variável eficiência de uso de nitrogênio segundo metodologia de Fischer et al. (1983), que não apresentou diferença significativa. Médici et al. (2004) avaliando eficiência de uso do nitrogênio também observou significância $(\mathrm{p}<0,01)$ pelo teste $\mathrm{F}$ para os genótipos, assim como também as características produtividade e peso de 100 sementes. Castro et al. (2009) avaliando genótipos de milho de alta qualidade proteica também observou diferenças significativas entre os genótipos para peso hectolítrico.

Já com relação ao nitrogênio, as características de produtividade e eficiência de uso do nitrogênio segundo metodologia de Moll et al. (1982) mostraram diferença significativa $(\mathrm{p}<0,01)$ pelo teste $\mathrm{F}$, assim como a característica do peso hectolítrico, com diferença significativa $(p<0,05)$ (TAB. 1). Médici et al. (2004) também relata significância para a eficiência de uso do nitrogênio assim como Fernandes et al. (2005) relatam significância para produtividade. $O$ peso de 100 sementes não apresentou diferença significativa com relação aos ambientes alto e baixo N, Costa (2000) relatou o mesmo efeito avaliando três doses de $\mathrm{N}$.

Tabela 1 - Resumo da análise de variância conjunta de peso de 100 sementes (P100S), peso hectolítrico (PH) e produtividade (Prod), eficiência de uso do nitrogênio segundo Moll et al. (1982) e segundo Fischer et al. (1983) de 24 populações de milho e uma cultivar como testemunha (BR106) em Gurupi - TO, safra 2009/2010

\begin{tabular}{ccccccc}
\hline \multirow{2}{*}{ F.V. } & G.L & \multicolumn{5}{c}{ QM } \\
\cline { 2 - 6 } & & P100S & PH & Prod & Moll & Fischer $^{1}$ \\
\hline População & 24 & $26,6536^{* *}$ & $27,106^{* *}$ & $1899827^{* *}$ & $1068,5^{* *}$ & $0,0132^{\mathrm{ns}}$ \\
F (Pop) & - & 6,067 & 4,283 & 3,268 & 2,443 & 0,776 \\
N & 1 & $1,2769^{\mathrm{ns}}$ & $30,691^{*}$ & $30181838^{* *}$ & $645644^{* *}$ & - \\
F (N) & - & 0,291 & 4,840 & 51,91 & 1476 & - \\
N*Pop & 24 & $4,0450^{\mathrm{ns}}$ & $8,310^{\mathrm{ns}}$ & $376116^{\mathrm{ns}}$ & $703,9^{\mathrm{ns}}$ & - \\
F(N*Pop) & - & 0,921 & 1,313 & 0,647 & 1,609 & - \\
Rep(N) & 2 & $8,2277^{\mathrm{ns}}$ & $6,722^{\mathrm{ns}}$ & $333716,3^{\mathrm{ns}}$ & $570,8^{\mathrm{ns}}$ & $0,0131^{\mathrm{ns}}$ \\
Erro & 48 & 4,3933 & 6,328 & 581587,8 & 437,3 & 0,017 \\
C.V. $(\%)$ & - & 7,4 & 4,2 & 14,6 & 18,4 & 19,6 \\
\hline
\end{tabular}

* e ** significativo a 0,05 e 0,01 de probabilidade respectivamente pelo teste $\mathrm{F}$; ${ }^{\text {ns }}$, não significativo; ${ }^{1}$ Para EUN segundo Fischer, análise de variância apenas para genótipos, GL de Bloc $=1$ e Erro $=24$ 
Para a interação populações $\mathrm{x}$ nitrogênio, não houve significância para qualquer característica (TAB. 1), concordando com Médici et al. (2004) que relataram o mesmo efeito para produtividade, peso de 100 sementes e eficiência de uso do nitrogênio. Fernandes e Buzetti (2005) avaliando genótipos comerciais de milho também não encontraram diferença significativa para interação genótipos x nitrogênio.

A variável peso hectolítrico (TAB. 2) apresentou média das populações em alto $\mathrm{N}$ significativamente inferior em relação à média das populações em baixo $\mathrm{N}$,
58,6 e $59,7 \mathrm{~kg} \mathrm{haL}^{-1}$, respectivamente. O mesmo efeito foi observado para a população 25-1 que mostrou-se inferior em alto N. Coelho et al. (1998) trabalhando trigo, observaram que o peso hectolítrico reduziu linearmente com o incremento das doses de $\mathrm{N}$ em dois anos, assim como Trindade et al. (2006) observaram o mesmo efeito, concluindo que o peso hectolítrico diminui linearmente com o aumento das doses de nitrogênio. Estes autores ainda relatam outros casos na literatura onde ocorreram o mesmo efeito e atribuíram tal fenômeno ao aumento do número de grãos por espiga e maior competição entre

Tabela 2 - Valores médios de peso hectolítrico em $\mathrm{kg} \mathrm{hL}^{-1}(\mathrm{PH})$ e peso de 100 sementes em g (P100S) de 24 populações de milho e uma variedade comercial, BR 106, usada como testemunha, em alta e baixa disponibilidade de N em Gurupi - TO, safra 2009/2010

\begin{tabular}{|c|c|c|c|c|c|c|}
\hline \multirow{2}{*}{ População } & \multicolumn{3}{|l|}{$\mathrm{PH}$} & \multicolumn{3}{|l|}{ P100S } \\
\hline & Alto $\mathrm{N}$ & Baixo N & Média & Alto N & Baixo N & Média \\
\hline $1-3$ & $56,3 \mathrm{bA}$ & 58,6 aA & $57,4 \mathrm{~b}$ & 33,9 aA & $34,1 \mathrm{aA}$ & $34,0 \mathrm{a}$ \\
\hline $10-6$ & $57,9 \mathrm{bA}$ & $59,4 \mathrm{aA}$ & $58,7 \mathrm{~b}$ & $31,9 \mathrm{aA}$ & $32,4 \mathrm{aA}$ & $32,1 \mathrm{a}$ \\
\hline $10-1$ & $60,7 \mathrm{aA}$ & 58,6 aA & $59,6 \mathrm{a}$ & $30,7 \mathrm{aA}$ & $30,7 \mathrm{aA}$ & $30,7 \mathrm{a}$ \\
\hline $12-6$ & $58,5 \mathrm{bA}$ & $62,2 \mathrm{aA}$ & $60,3 \mathrm{a}$ & $31,0 \mathrm{aA}$ & $30,2 \mathrm{aA}$ & $30,6 \mathrm{a}$ \\
\hline P.O. & $56,0 \mathrm{bA}$ & $59,8 \mathrm{aA}$ & $57,9 \mathrm{~b}$ & $30,9 \mathrm{aA}$ & $29,7 \mathrm{aA}$ & $30,3 \mathrm{a}$ \\
\hline $12-5$ & $60,5 \mathrm{aA}$ & $58,3 \mathrm{aA}$ & $59,4 \mathrm{~b}$ & $29,6 \mathrm{aA}$ & 30,9 aA & $30,3 \mathrm{a}$ \\
\hline $25-2$ & $56,4 \mathrm{bA}$ & $57,9 \mathrm{aA}$ & $57,1 \mathrm{~b}$ & $29,5 \mathrm{aA}$ & $29,8 \mathrm{aA}$ & $29,6 \mathrm{a}$ \\
\hline $12-2$ & $58,5 \mathrm{bA}$ & $58,1 \mathrm{aA}$ & $58,3 \mathrm{~b}$ & $29,3 \mathrm{aA}$ & $30,0 \mathrm{aA}$ & $29,6 \mathrm{a}$ \\
\hline $11-3$ & $58,5 \mathrm{bA}$ & $59,6 \mathrm{aA}$ & $59,1 \mathrm{~b}$ & $30,8 \mathrm{aA}$ & $28,1 \mathrm{aA}$ & $29,5 \mathrm{a}$ \\
\hline $12-4$ & $60,9 \mathrm{aA}$ & $57,5 \mathrm{aA}$ & $59,2 \mathrm{~b}$ & $28,0 \mathrm{bA}$ & $30,7 \mathrm{aA}$ & $29,3 \mathrm{a}$ \\
\hline $2-5$ & $62,9 \mathrm{aA}$ & $58,1 \mathrm{aA}$ & $60,5 \mathrm{a}$ & $29,4 \mathrm{aA}$ & $28,9 \mathrm{aA}$ & $29,2 \mathrm{a}$ \\
\hline BR106 & $61,2 \mathrm{aA}$ & $62,0 \mathrm{aA}$ & $61,6 \mathrm{a}$ & $29,6 \mathrm{aA}$ & $27,2 \mathrm{bA}$ & $28,4 \mathrm{a}$ \\
\hline $25-1$ & $50,5 \mathrm{cB}$ & $58,8 \mathrm{aA}$ & $54,6 \mathrm{c}$ & $31,3 \mathrm{aA}$ & $25,5 \mathrm{bB}$ & $28,4 \mathrm{a}$ \\
\hline $28-5$ & $58,0 \mathrm{bA}$ & 57,9 aA & $57,9 \mathrm{~b}$ & $27,2 \mathrm{bA}$ & $29,2 \mathrm{aA}$ & $28,2 \mathrm{a}$ \\
\hline $35-5$ & $55,7 \mathrm{bA}$ & $55,4 \mathrm{aA}$ & $55,5 \mathrm{c}$ & $27,6 \mathrm{bA}$ & $28,4 \mathrm{aA}$ & $28,0 \mathrm{a}$ \\
\hline $12-3$ & $58,8 \mathrm{bA}$ & $61,0 \mathrm{aA}$ & $59,9 \mathrm{a}$ & $28,4 \mathrm{aA}$ & $26,7 \mathrm{bA}$ & $27,5 \mathrm{~b}$ \\
\hline $25-5$ & $51,0 \mathrm{cA}$ & $55,6 \mathrm{aA}$ & $53,3 \mathrm{c}$ & $27,4 \mathrm{bA}$ & $26,8 \mathrm{bA}$ & $27,1 \mathrm{~b}$ \\
\hline $32-3$ & $57,0 \mathrm{bA}$ & $58,6 \mathrm{aA}$ & $57,8 \mathrm{~b}$ & $26,2 \mathrm{bA}$ & $27,8 \mathrm{aA}$ & $27,0 \mathrm{~b}$ \\
\hline $1-5$ & $55,8 \mathrm{bA}$ & $59,2 \mathrm{aA}$ & $57,5 \mathrm{~b}$ & $26,0 \mathrm{bA}$ & $27,9 \mathrm{aA}$ & $27,0 \mathrm{~b}$ \\
\hline $26-1$ & $61,7 \mathrm{aA}$ & $63,8 \mathrm{aA}$ & $62,8 \mathrm{a}$ & $25,6 \mathrm{bA}$ & $27,8 \mathrm{aA}$ & $26,7 \mathrm{~b}$ \\
\hline $15-1$ & $56,6 \mathrm{bA}$ & $60,4 \mathrm{aA}$ & $58,5 \mathrm{~b}$ & $26,4 \mathrm{bA}$ & $26,2 \mathrm{bA}$ & $26,3 \mathrm{~b}$ \\
\hline $15-2$ & $64,0 \mathrm{aA}$ & $60,5 \mathrm{aA}$ & $62,2 \mathrm{a}$ & $25,7 \mathrm{bA}$ & $26,7 \mathrm{bA}$ & $26,2 \mathrm{~b}$ \\
\hline $26-2$ & $63,5 \mathrm{aA}$ & $64,4 \mathrm{aA}$ & $63,9 \mathrm{a}$ & $24,2 \mathrm{bA}$ & $23,0 \mathrm{cA}$ & $23,6 \mathrm{c}$ \\
\hline $30-3$ & $61,4 \mathrm{aA}$ & $61,7 \mathrm{aA}$ & $61,6 \mathrm{a}$ & $25,6 \mathrm{bA}$ & $21,4 \mathrm{cA}$ & $23,5 \mathrm{c}$ \\
\hline $15-3$ & $62,1 \mathrm{aA}$ & $64,5 \mathrm{aA}$ & $63,3 \mathrm{a}$ & $23,0 \mathrm{bA}$ & $23,5 \mathrm{cA}$ & $23,2 \mathrm{c}$ \\
\hline Média & $58,6 \mathrm{~B}$ & $59,7 \mathrm{~A}$ & 59,2 & $28,4 \mathrm{~A}$ & $28,1 \mathrm{~A}$ & 28,2 \\
\hline
\end{tabular}

Médias seguidas de mesma letra não diferem entre si pelo teste de Scott-Knott, ao nível de 0,05 de probabilidade. Letras minúsculas para comparação na coluna e letras maiúsculas para comparação na linha dentro da mesma característica. 
os grãos por fotoassimilados, uma vez que o aumento da dose de nitrogênio aumenta o número de grãos por unidade de área.

Kolchinski e Schuch (2004) também observaram menores pesos hectolítrico com o aumento das doses de nitrogênio em aveia, justificando tal efeito pelo maior gasto de energia da transformação de proteínas nas sementes, que ocorre em plantas com maiores disponibilidade de $\mathrm{N}$, já que em menores disponibilidades de nitrogênio as sementes de cereais tentem a acumular carboidratos.

Mallmann et al. (1994) trabalhando com populações segregantes de trigo, afirmam que grãos menores proporcionam melhor ajuste no cilindro, evitando espaços vazios e provocando maior aglutinação dos grãos no momento de aferição do peso hectolítrico, podendo-se também, através deste fato, justificar o maior peso hectolítrico em menores disponibilidade de nitrogênio no solo.

As populações em baixo $\mathrm{N}$ não diferiram pelo teste de Scott-Knott (1974), já em alto $\mathrm{N}$ as populações se dividiram em três grupos (TAB. 2), o grupo dos maiores valores de peso hectolítrico com médias variando de 64,0 a $60,5 \mathrm{~kg} \mathrm{hL}^{-1}$ com 9 populações e a testemunha BR106, o grupo intermediário com 13 populações e médias variando de 58,8 a $55,7 \mathrm{~kg} \mathrm{hL}^{-1}$ e o grupo de menores valores, com apenas duas populações, cujas médias foram 51,0 e $50,5 \mathrm{~kg} \mathrm{hL}^{-1}$.

As médias das populações independentemente do ambiente foram classificadas em três grupos, com 9 populações e a testemunha BR106 no grupo superior, com médias variando de 63,9 a $59,6 \mathrm{~kg} \mathrm{hL}^{-1}, 12$ populações no grupo intermediário, com médias variando de 59,4 a $57,1 \mathrm{~kg} \mathrm{hL}^{-1}$ e três populações no grupo inferior, com médias variando de 55,5 a 53,3 $\mathrm{kg} \mathrm{hL}^{-1}$ (TAB. 2).

Castro et al. (2009) também observaram diferenças entre os genótipos com relação ao peso hectolítrico, e citaram que a densidade do grão está comumente associada à dureza, sendo que a dureza do endosperma interfere, diretamente, no rendimento e no valor agregado de produtos derivados do milho. Trindade et al. (2006) ressalta a importância desta variável, pois serve como parâmetro de qualidade para a comercialização de grãos.

Segundo Marcos Filho et al. (1987) o peso hectolítrico é uma característica varietal, e varia de acordo com as condições de clima e solo, adubação, maturidade da semente, incidência de pragas e doenças, grau de umidade entre outras, concluindo que as sementes de melhor qualidade são aquelas que obtiverem maiores pesos hectolítrico, concordando com Carvalho e Nakagawa (2000) que afirmam que sementes maiores e de maiores densidades possuem melhor qualidade, apresentando maior vigor.
Segundo Marcos Filho et al. (1987) o peso de 100 sementes é um dado importante que pode nos dar ideia a respeito da qualidade das sementes, bem como fornecer informações que facilitam o cálculo para semeadura e regulagens de semeadeiras. Para a característica de peso de 100 sementes (TAB. 2) não foi observado diferenças significativas nas médias entre alto e baixo $\mathrm{N}$, e somente a população 25-1 mostrou-se significativamente inferior em baixo N. Esta mesma população apresentou diferenças significativas entre alto e baixo $\mathrm{N}$ para o peso hectolítrico, porém sendo inferior em alto $\mathrm{N}$.

Em alto $\mathrm{N}$ as populações se dividiram em dois grupos, sendo 12 populações e a testemunha no grupo superior e 12 no grupo inferior, com médias variando para o grupo superior de 33,9 a 28,4 g e no grupo inferior de 28,0 a 23,0 g. Já em baixo $\mathrm{N}$ as populações se dividiram em três grupos, com 16 populações no grupo superior com médias variando de 34,1 a $27,8 \mathrm{~g}, 5$ populações e a testemunha BR106 no grupo intermediário, com médias variando de 27,2 a 25,5 g e três populações compondo o grupo inferior com médias variando de 23,5 a 21,4 g (TAB. 2).

De forma semelhante ao baixo $\mathrm{N}$, a média das populações independente do ambiente também foi classificada em três grupos estatísticos, com 14 populações e a testemunha BR106 presente no grupo superior, com médias variando de 34,0 a 28,0 g. Sete populações integram o grupo intermediário, com médias variando de 27,5 a 26,2 g e três no grupo inferior, com médias variando de 23,6 a 23,2 g (TAB. 2) podendo atribuir este efeito às características dos genótipos. Fageria et al. (2007) afirmam que o peso de 100 sementes é um componente da produtividade bastante estável e característico da cultivar, podendo portanto ser utilizado na seleção indireta para o aumento da produtividade, já que este sofre menor interferência do fator ambiente.

Coelho et al. (1998) atribuíram ao aumento nos valores da massa de cem grãos com o aumento no número de grãos por espiga, diminuindo a competição por nutrientes e fotoassimilados dentro da espiga e, como consequência, aumentando a massa dos grãos. Carvalho e Nakagawa (2000) afirmaram que sementes de maior tamanho são as que normalmente foram melhor nutridas durante seu desenvolvimento, apresentando também maiores densidades, por este motivo, estas são as que possuem normalmente embriões bem formados, maiores quantidades de reserva e potencialmente mais vigorosas. Concordando com Andrade et al. (2001) onde citaram que as sementes maiores são geralmente reportadas como sendo de melhor qualidade e, por isso, preferencialmente utilizadas pelos agricultores, bem como os autores Shieh e McDonald (1982) que verificaram maiores produtividades quando são utilizadas sementes maiores. 
A produtividade (TAB. 3) foi significativamente maior em alto $\mathrm{N}$ com relação a baixo $\mathrm{N}$, com média de 5.748 e $4.649 \mathrm{~kg} \mathrm{ha}^{-1}$, respectivamente, sendo o baixo $\mathrm{N}$ $23 \%$ menos produtivo em relação a alto N. Souza (2007) avaliando famílias de meios-irmãos de milho observou $32 \%$ de aumento de produtividade no ambiente de alto N. No ambiente de alto N, 18 populações apresentamse no grupo de produtividades superiores, sendo que a população mais produtiva foi a $12-6$, com $6.956 \mathrm{~kg} \mathrm{ha}^{-1}$ e a população 11-3 a menos produtiva neste grupo, com média de $5.500 \mathrm{~kg} \mathrm{ha}^{-1}$. No grupo inferior, agrupou-se 6 populações e a testemunha BR106, cujas médias variaram de 5.221 a $4.001 \mathrm{~kg} \mathrm{ha}^{-1}$.
Já em baixo $\mathrm{N}$ as populações não diferiram quanto à produtividade, mesmo com as médias variando de 6.019 a $3.589 \mathrm{~kg} \mathrm{ha}^{-1}$, isto pode ser explicado devido à expressão da variabilidade genética ser inferior em ambientes limitantes, como o estresse pela falta de nitrogênio. Souza (2007) observou menor expressão da variabilidade genética de uma população de polinização aberta de milho em ambiente estressante quanto à disponibilidade de nitrogênio, corroborando com os dados encontrados neste experimento.

Mesmo o ambiente em baixo $\mathrm{N}$ mostrando significativamente inferior, a média encontrada, $4.649 \mathrm{~kg} \mathrm{ha}^{-1}$ (TAB. 3), está acima da média estimada

Tabela 3 - Valores médios de produtividade $\left(\mathrm{kg} \mathrm{ha}^{-1}\right)$ de 24 populações de milho e uma cultivar testemunha (BR106) em alta e baixa disponibilidade de N em Gurupi - TO, safra 2009/2010

\begin{tabular}{|c|c|c|c|}
\hline População & Alto $\mathrm{N}$ & Baixo N & Média \\
\hline $12-6$ & $6956 \mathrm{aA}$ & $5740 \mathrm{aA}$ & $6348 \mathrm{a}$ \\
\hline $25-5$ & $6751 \mathrm{aA}$ & $5940 \mathrm{aA}$ & $6346 a$ \\
\hline $35-5$ & $6437 \mathrm{aA}$ & $6019 \mathrm{aA}$ & $6228 \mathrm{a}$ \\
\hline $12-2$ & $6880 \mathrm{aA}$ & $5418 \mathrm{aA}$ & $6149 a$ \\
\hline $12-3$ & $6525 \mathrm{aA}$ & $5348 \mathrm{aA}$ & $5936 \mathrm{a}$ \\
\hline $1-5$ & $6436 \mathrm{aA}$ & $5113 \mathrm{aA}$ & $5774 \mathrm{a}$ \\
\hline $10-6$ & $5955 \mathrm{aA}$ & $5141 \mathrm{aA}$ & $5548 \mathrm{~b}$ \\
\hline $25-2$ & $6020 \mathrm{aA}$ & $4844 \mathrm{aA}$ & $5432 \mathrm{~b}$ \\
\hline $1-3$ & $5877 \mathrm{aA}$ & $4901 \mathrm{aA}$ & $5389 \mathrm{~b}$ \\
\hline $28-5$ & $6222 \mathrm{aA}$ & $4447 \mathrm{aB}$ & $5334 \mathrm{~b}$ \\
\hline $11-3$ & $5500 \mathrm{aA}$ & $5141 \mathrm{aA}$ & $5321 \mathrm{~b}$ \\
\hline $12-4$ & $6180 \mathrm{aA}$ & $4202 \mathrm{aB}$ & $5191 \mathrm{~b}$ \\
\hline $10-1$ & $5617 \mathrm{aA}$ & $4723 \mathrm{aA}$ & $5170 \mathrm{~b}$ \\
\hline $12-5$ & $5918 \mathrm{aA}$ & $4408 \mathrm{aA}$ & $5163 \mathrm{~b}$ \\
\hline P.O. & $5706 \mathrm{aA}$ & $4613 \mathrm{aA}$ & $5159 \mathrm{~b}$ \\
\hline $25-1$ & $6624 \mathrm{aA}$ & $3635 \mathrm{aB}$ & $5129 \mathrm{~b}$ \\
\hline $15-1$ & $5530 \mathrm{aA}$ & $4608 \mathrm{aA}$ & $5069 \mathrm{~b}$ \\
\hline $26-1$ & $5590 \mathrm{aA}$ & $4313 \mathrm{aA}$ & $4951 \mathrm{c}$ \\
\hline $32-3$ & $5080 \mathrm{bA}$ & $4433 \mathrm{aA}$ & $4756 \mathrm{c}$ \\
\hline $15-2$ & $5221 \mathrm{bA}$ & $3719 \mathrm{aA}$ & $4470 \mathrm{c}$ \\
\hline BR106 & $4880 \mathrm{bA}$ & $4035 \mathrm{aA}$ & $4458 \mathrm{c}$ \\
\hline $26-2$ & $4807 \mathrm{bA}$ & $3760 \mathrm{aA}$ & $4283 \mathrm{c}$ \\
\hline $30-3$ & $4413 \mathrm{bA}$ & $3937 \mathrm{aA}$ & $4175 \mathrm{c}$ \\
\hline $2-5$ & $4001 \mathrm{bA}$ & $4213 \mathrm{aA}$ & $4107 \mathrm{c}$ \\
\hline $15-3$ & $4583 \mathrm{bA}$ & $3589 \mathrm{aA}$ & $4086 \mathrm{c}$ \\
\hline Média & $5748 \mathrm{~A}$ & $4649 \mathrm{~B}$ & 5198,5 \\
\hline
\end{tabular}

Médias seguidas de mesma letra, minúsculas para comparação na coluna e letras maiúsculas para comparação na linha não diferem entre si pelo teste de Scott-Knott, ao nível de 0,05 de probabilidade 
para o estado do Tocantins, safra 2009/2010, que é de $2.850 \mathrm{~kg} \mathrm{ha}^{-1}$ (CONAB, 2010), o que indica que as populações avaliadas possuem certa tolerância ao ambiente de baixa disponibilidade de nitrogênio no solo, com exceção apenas das populações $25-1$; 28 5 e 12-4, que foram as únicas a apresentar redução significativa da produtividade em baixo $\mathrm{N}$, podendo então estas populações serem mais responsivas a ambientes com maiores disponibilidade de nitrogênio. Médici et al. (2004) afirmaram que a seleção para performance sob baixo $\mathrm{N}$ em germoplasma de milho parece melhorar a eficiência com que o $\mathrm{N}$ é utilizado para produzir biomassa e grãos, tanto em ambientes de baixa disponibilidade de nitrogênio quanto em alta disponibilidade.

As populações $12-6 ; 25-5 ; 35-5 ; 12-2 ; 12-$ 3 e $1-5$ formam o grupo das populações de maiores produtividades independente da condição de $\mathrm{N}$ no solo, com médias variando de 6.348 a $5.774 \mathrm{~kg} \mathrm{ha}^{-1}$. No grupo intermediário observa-se 11 populações com médias variando de 5.548 a $5.069 \mathrm{~kg} \mathrm{ha}^{-1}$ e no grupo de produtividades inferiores encontram-se sete populações, e a testemunha BR106 com médias de 4.951 a $4.086 \mathrm{~kg} \mathrm{ha}^{-1}$ (TAB. 3).

Segundo Valentin et al. (2005) o nitrogênio é absorvido pelas plantas na forma de nitrato e é reduzido à amônia, possibilitando assim sua assimilação. No processo redutivo o nitrito é reduzido à amônia e assimilado na forma orgânica resultando na síntese de aminoácidos e posteriormente proteínas, clorofila e outros. Portanto as condições de deficiência de nitrogênio resultaram em menor produção de clorofila e proteínas, que implicará em menor produção de fotoassimilados, assim em menores produtividades.

A eficiência de uso de nitrogênio segundo Moll et al. (1982) (TAB. 4) não se diferiu entre as populações no ambiente de alto $\mathrm{N}$, e apresentou uma média de 33,0 indicando que para cada $\mathrm{kg}$ de $\mathrm{N}$ aplicado no solo a planta produziu $33 \mathrm{~kg}$ de grãos. Já quando comparamos as populações entre alto e baixo $\mathrm{N}$ observamos que para todas as populações o ambiente de baixo $\mathrm{N}$ apresentouse superior estatisticamente, evidenciando que para melhor eficiência e uso de nitrogênio pode-se reduzir a adubação nitrogenada a níveis que ainda proporcione produtividades satisfatórias (MAJEROWICZ et al., 2002; MÉDICI et al., 2004). Concordando com o presente estudo, Fernandes et al. (2005) relataram o mesmo efeito avaliando híbridos de milho.

Com relação à média das populações para a eficiência de uso de nitrogênio, independente dos ambientes, observa-se dois grupos, com 10 populações no grupo mais eficiente na utilização de nitrogênio sendo elas $35-5 ; 25-5 ; 12-6 ; 12-2 ; 10-1 ; 1-5 ; 10-6 ; 11-3 ; 1-3$ e 25-2, apresentando índices de 143,9 a 143,1, e no grupo dos menos eficientes encontraram-se 14 populações e a testemunha BR106 com índices variando de 114,5 a 87,9, sendo a testemunha com 98 (TAB. 4).

Fernandes et al. (2005) avaliando a eficiência de uso de nitrogênio segundo a metodologia de Moll et al. (1982) encontraram valores semelhantes ao presente trabalho, com índices variando de 21,8 a 39,1 utilizando $180 \mathrm{~kg} \mathrm{ha}^{-1}$ de $\mathrm{N}$, enquanto neste estudo foi obtido uma variação de 23,0 a 39,9 com aplicação de $174 \mathrm{~kg} \mathrm{ha}^{-1}$ de $\mathrm{N}$.

McCullough et al. (1994), estudando materiais contrastantes quanto à eficiência de uso do nitrogênio, observaram que o genótipo mais sensível à deficiência de $\mathrm{N}$ foi o que apresentou maior diferença entre os níveis de N. Tal efeito pode ser observado através do índice de Fischer et al. (1983) já que para obtenção do índice é levado em conta a diferença entre os ambientes de alto e de baixo $\mathrm{N}$.

Segundo a metodologia de eficiência de uso de nitrogênio proposto por Fischer et al. (1983) não foi possível identificar as populações estatisticamente mais eficientes (TAB. 4), no entanto a população 25 apresentou-se $31,8 \%$ mais eficiente que a média deste índice. As populações $35-5$; 25-5; 10-6 e 11-3 mesmo não apresentando diferença entre as demais populações com relação ao índice de Fischer et al. (1983) apresentaram superioridade estatística no índice de Moll et al. (1982), sendo consideradas populações eficientes quanto ao uso de nitrogênio.

Assim, diferentes métodos de obtenção de novas cultivares comerciais podem ser exploradas a partir destas populações, visando o desenvolvimento de genótipos com maior eficiência de uso de nitrogênio para ambientes com e sem estresses dovrferido nutriente.

Para a correlação de Spearmam (TAB. 5) foi observado significância estatística a 0,01 de probabilidade de produção de grão com peso de 100 sementes, peso hectolítrico e índice de Moll, podendo estas variáveis ser utilizadas para seleção indireta para o aumento da produtividade, especialmente o peso de 100 sementes. No entanto, de acordo com Fageria et al. (2007), esta é uma característica geralmente estável e que sofre menor interferência do ambiente.

O índice de Moll et al. (1982) correlacionou-se significativamente com todas as características, com exceção apenas do índice de Fischer et al. (1983), sendo que este índice não mostrou correlação com qualquer característica avaliada. 
Tabela 4 - Valores médios da eficiência de uso de nitrogênio segundo Moll et al. (1982) e Fischer et al. (1983) de 25 populações de milho em Alto e Baixo N em Gurupi - TO, safra 2009/2010

\begin{tabular}{|c|c|c|c|c|c|}
\hline \multirow{2}{*}{ População } & \multicolumn{2}{|c|}{ Moll et al. (1982) } & \multirow[b]{2}{*}{ Média } & \multicolumn{2}{|c|}{ Fischer et al. (1983) } \\
\hline & Alto $\mathrm{N}$ & Baixo N & & Índice & $\%$ \\
\hline $2-5$ & $23,0 \mathrm{aB}$ & $175,5 \mathrm{bA}$ & $99,2 \mathrm{~b}$ & $0,87 \mathrm{a}$ & 131,8 \\
\hline $11-3$ & $31,6 \mathrm{aB}$ & $214,2 \mathrm{aA}$ & $122,9 \mathrm{a}$ & $0,77 \mathrm{a}$ & 116,7 \\
\hline $35-5$ & $37,0 \mathrm{aB}$ & $250,8 \mathrm{aA}$ & $143,9 \mathrm{a}$ & $0,76 \mathrm{a}$ & 115,2 \\
\hline $30-3$ & $25,4 \mathrm{aB}$ & $164,0 \mathrm{bA}$ & $94,7 \mathrm{~b}$ & $0,72 \mathrm{a}$ & 109,1 \\
\hline $32-3$ & $29,2 \mathrm{aB}$ & $184,7 \mathrm{bA}$ & $106,9 \mathrm{~b}$ & $0,71 \mathrm{a}$ & 107,6 \\
\hline $25-5$ & $38,8 \mathrm{aB}$ & $247,5 \mathrm{aA}$ & $143,1 \mathrm{a}$ & $0,71 \mathrm{a}$ & 107,6 \\
\hline $10-6$ & $34,2 \mathrm{aB}$ & $214,2 \mathrm{aA}$ & $124,2 \mathrm{a}$ & $0,69 \mathrm{a}$ & 104,5 \\
\hline $10-1$ & $32,3 \mathrm{aB}$ & $196,8 \mathrm{bA}$ & $114,5 \mathrm{~b}$ & $0,69 \mathrm{a}$ & 104,5 \\
\hline $12-6$ & $39,9 \mathrm{aB}$ & $239,1 \mathrm{aA}$ & $139,5 \mathrm{a}$ & $0,68 \mathrm{a}$ & 103,0 \\
\hline $\mathrm{PO}$ & $32,8 \mathrm{aB}$ & $192,2 \mathrm{bA}$ & $112,5 \mathrm{~b}$ & $0,67 \mathrm{a}$ & 101,5 \\
\hline BR106 & $28,0 \mathrm{aB}$ & $168,1 \mathrm{bA}$ & $98,0 \mathrm{~b}$ & $0,67 \mathrm{a}$ & 101,5 \\
\hline $15-1$ & $31,8 \mathrm{aB}$ & $192,0 \mathrm{bA}$ & $111,9 \mathrm{~b}$ & $0,67 \mathrm{a}$ & 101,5 \\
\hline $1-3$ & $33,7 \mathrm{aB}$ & $204,2 \mathrm{aA}$ & $119,0 \mathrm{a}$ & $0,67 \mathrm{a}$ & 101,5 \\
\hline $1-5$ & $37,0 \mathrm{aB}$ & $213,0 \mathrm{aA}$ & $125,0 \mathrm{a}$ & $0,67 \mathrm{a}$ & 101,5 \\
\hline $25-2$ & $34,6 \mathrm{aB}$ & $201,8 \mathrm{aA}$ & $118,2 \mathrm{a}$ & $0,66 \mathrm{a}$ & 100,0 \\
\hline $12-3$ & $37,5 \mathrm{aB}$ & $222,8 \mathrm{aA}$ & $130,1 \mathrm{a}$ & $0,66 \mathrm{a}$ & 100,0 \\
\hline $15-3$ & $26,3 \mathrm{aB}$ & $149,5 \mathrm{bA}$ & $87,9 \mathrm{~b}$ & $0,65 \mathrm{a}$ & 98,5 \\
\hline $12-2$ & $39,5 \mathrm{aB}$ & $225,7 \mathrm{aA}$ & $132,6 \mathrm{a}$ & $0,64 \mathrm{a}$ & 97,0 \\
\hline $26-2$ & $27,6 \mathrm{aB}$ & $156,6 \mathrm{bA}$ & $92,1 \mathrm{~b}$ & $0,64 \mathrm{a}$ & 97,0 \\
\hline $26-1$ & $32,1 \mathrm{aB}$ & $179,7 \mathrm{bA}$ & $105,9 \mathrm{~b}$ & $0,62 \mathrm{a}$ & 93,9 \\
\hline $12-5$ & $34,0 \mathrm{aB}$ & $183,6 \mathrm{bA}$ & $108,8 \mathrm{~b}$ & $0,60 \mathrm{a}$ & 90,9 \\
\hline $15-2$ & $30,0 \mathrm{aB}$ & $155,0 \mathrm{bA}$ & $92,5 \mathrm{~b}$ & $0,58 \mathrm{a}$ & 87,9 \\
\hline $28-5$ & $35,7 \mathrm{aB}$ & $185,2 \mathrm{bA}$ & $110,5 \mathrm{~b}$ & $0,57 \mathrm{a}$ & 86,4 \\
\hline $12-4$ & $35,5 \mathrm{aB}$ & $175,1 \mathrm{bA}$ & $105,3 \mathrm{~b}$ & $0,55 \mathrm{a}$ & 83,3 \\
\hline $25-1$ & $38,0 \mathrm{aB}$ & $151,4 \mathrm{bA}$ & $94,7 \mathrm{~b}$ & $0,44 \mathrm{a}$ & 66,7 \\
\hline Média & $33,0 \mathrm{~B}$ & $193,7 \mathrm{~A}$ & 113,4 & 0,66 & 100,0 \\
\hline
\end{tabular}

Médias seguidas de mesma letra, minúsculas para comparação na coluna e letras maiúsculas para comparação na linha não diferem entre si pelo teste de Scott-Knott, ao nível de 0,05 de probabilidade

Tabela 5 - Valores da correlação de Spearman para peso de 100 sementes (P100S), peso hectolítrico (PH), produtividade (Prod), índice de Moll et al. (1982) e índice de Fischer et al. (1983) de 24 populações de milho em Gurupi - TO, safra 2009/2010

\begin{tabular}{clccc}
\hline Característica & P100S & PH & Prod & Moll \\
\hline PH & $-0,329$ & - & - & - \\
Prod & $0,520^{* *}$ & $-0,634^{* *}$ & - & - \\
Moll & $0,517^{* *}$ & $-0,586^{* *}$ & $0,928^{* *}$ & - \\
Fischer & $-0,259$ & $-0,270$ & 0,031 & 0,215 \\
\hline
\end{tabular}

** significativo a 0,01 de probabilidade pelo teste $\mathrm{t}$ 


\section{Conclusões}

1. Pela metodologia de Moll et al. (1982) é possível diferenciar as populações quanto à eficiência no uso de nitrogênio no ambiente de baixa disponibilidade de nitrogênio no solo;

2. As características estudadas foram influenciadas pelos diferentes níveis de nitrogênio aplicado, exceto para peso de 100 sementes;

3. As populações $2-5 ; 35-5 ; 25-5 ; 12-6 ; 12-2 ; 10-$ $1 ; 1-5 ; 10-6$ e 11-3 apresentam potencial para serem exploradas como fonte de germoplasma visando à obtenção de genótipos tolerantes a baixas disponibilidades de nitrogênio;

4. As populações avaliadas apresentaram diferenças entre si em todas as características estudadas, indicando variabilidade;

5. O índice de Moll et al. (1982) apresenta associação significativa com todas as características estudadas exceto para o índice de Fischer et al. (1983) que indica ser possível a obtenção de genótipos eficientes no uso de nitrogênio por meio de seleção destas características.

\section{Agradecimentos}

À CAPES pela concessão de bolsa ao primeiro autor e ao CNPq pelo financiamento do projeto.

\section{Referências}

ANDRADE, R. V. et al. Qualidade fisiológica das sementes do milho híbrido simples HS 200 em relação ao tamanho. Revista Ciência e Agrotecnologia, v. 25, n. 03, p. 576-582, 2001.

BREDEMEIER, C.; MUNDSTOCK, C. Regulação da absorção e assimilação do nitrogênio nas plantas. Ciência Rural, v. 30, n. 02 , p. 365-372, 2000.

CARVALHO, N. M.; NAKAGAWA, J. Sementes: ciência, tecnologia e produção. Funep, Jaboticabal, SP, 2000. 588 p.

CARVALHO, H. W. L.; SOUZA, E. M. Ciclos de seleção de progênies de meios-irmãos do milho BR 5011 Sertanejo. Pesquisa Agropecuária Brasileira, v. 42, n. 06, p. 803-809, 2007.

CASTRO, M. V. L. et al. Rendimento industrial e composição química de milho de alta qualidade proteica em relação a híbridos comerciais. Pesquisa Agropecuária Tropical, v. 39, n. 03, p. 233-242, 2009.

COELHO, M. A. O. et al. Resposta da produtividade de grãos e outras características agronômicas do trigo embrapa-22 irrigado ao nitrogênio em cobertura. Revista Brasileira de Ciências do Solo, v. 22, p. 555-561, 1998.

COIMBRA, R. R. et al. Caracterização e divergência genética de populações de milho resgatadas do Sudeste de Minas Gerais. Revista Ciência Agronômica, v. 41, n. 01, p. 159-166, 2010.

COMPANHIA NACIONAL DO ABASTECIMENTO (CONAB) Série histórica. Comparativo de área, produção e produtividade. Disponível em: <http://www.conab.gov.br/ conabweb/index.php?PAG=131>. Acesso em: 18 mai. 2010.

COSTA, A. M. Adubação nitrogenada na cultura do milho (Zea mays L.) em sistema de plantio direto. 2000. $90 \mathrm{f}$. Dissertação (Mestrado em Agronomia) - Faculdade de Ciências Agronômicas, Universidade Estadual de São Paulo, Botucatu.

FAGERIA, N. K.; SANTOS, A. B.; CUTRIM, V. A. Produtividade de arroz irrigado e eficiência de uso do nitrogênio influenciadas pela fertilização nitrogenada. Pesquisa Agropecuária Brasileira, v. 42, n. 07, p. 1029-1034, 2007.

FERNANDES, F. C. S.; BUZETTI, S. Efeito de níveis de nitrogênio na produtividade de seis cultivares de milho (Zea mays L.). Revista Científica Eletrônica de Agronomia, v. 07, n. $01,2005$.

FERNANDES, F. C. S. et al. Doses, eficiência e uso de nitrogênio por seis cultivares de milho. Revista Brasileira de Milho e Sorgo, v. 04, n. 02, p. 195-204, 2005.

FISCHER, K. S.; JOHNSON, E. C.; EDMEADS, G. O. Breeding and selection for drought resistance in tropical maize. Mexico: CIMMYT, 1983. 16 p.

JAKELAITIS, A.; SILVA, A. A.; FERREIRA, L. R. Efeitos do nitrogênio sobre o milho cultivado em consórcio com Brachiaria brizantha. Acta Scientiarum Agronomy, v. 27, n. 01, p. 39-46, 2005.

KOLCHISNSKI, E. M. e SCHUCH, L. O. B. Relações entre a adubação nitrogenada e a qualidade de grãos e de sementes em aveia branca. Ciência Rural, v. 34, n. 02, p. 379-383, 2004.

MAJEROWICZ, N. et al. Estudo da eficiência de uso do nitrogênio em variedades locais e melhoradas de milho. Revista Brasileira de Botânica, v. 25, n. 02, p. 129-136, 2002.

MALLMANN, I. L. et al. Mecanismos de seleção aplicados sobre o caráter tamanho de grãos em populações segregantes de trigo. Pesquisa Agropecuária Brasileira, v. 29, n. 03, p. 427-437, 1994.

MARCOS FILHO, J.; CICERO, S. M.; SILVA, W. R. Avaliação da qualidade das sementes. Piracicaba: FEALQ, 1987. 230 p.

McCULLOUGH, D. E. et al. Infuence of $\mathrm{N}$ supply on development and dry matter accumulation of old and a new maize hibrid. Canadian Journal of Plant Science, v. 74, p. 471-474, 1994.

MÉDICI, L. O. et al. Diallel analysis of maize lines with contrasting responses to applied nitrogen. Journal of Agricultural Science, v. 142, n. 05, p. 535-541, 2004.

MOLL, R. H.; KAMPRATH, E. J.; JACKSON, W. A. Analysis and interpretation of factors which contribute to 
efficiency of nitrogen utilization. Agronomy Journal, v. 74, n. 03, p. 562-564, 1982.

ROESCH, L. F. et al. Identificação de cultivares de milho eficientes na absorção de nitrogênio e na associação com bactérias diazotróficas. Ciência Rural, v. 35, n. 04, p. 924-927, 2005.

SCOTT, A. e KNOTT, M. Cluster analysis method for grouping means in analysis of variance. Biometrics, Washington, v. 30, n. 03, p. 507-512, 1974.

SHIEH, W. J.; McDONALD, M. B. The influence of seed size, shape and treatment on inbred seed corn quality. Seed Science and Technology, v. 10, n. 02, p. 307-313, 1982.

SILVA, A. G. et al. Influência da população de plantas e do espaçamento entre linhas nos caracteres agronômicos do híbrido de milho P30K75 em Rio Verde, Goiás. Bioscience Journal, v. 24, n. 02, p. 89-96. 2008.
SOUZA, L. V. Melhoramento de milho para eficiência no uso de nitrogênio. 2007. 54 f. Tese - (Doutorado em Genética e Melhoramento) - Universidade Federal de Viçosa, Viçosa/MG.

SOUZA, L. V. et al. Genetic control of grain yield and nitrogen use efficiency in tropical maize. Pesquisa Agropecuária Brasileira, v. 43, n. 11, p. 1517-1523, 2008.

TRINDADE, M. G. et al. Nitrogênio e água como fatores de produtividade do trigo no cerrado. Revista Brasileira de Engenharia Agrícola e Ambiental, v. 10, n. 01, p. 24-29, 2006.

VALENTIN, L.; COELHO, F. C.; FERREIRA, M. S. Teor de nitrogênio foliar e produtividade de três cultivares de milho (Zea mays L.) submetidos às adubações nitrogenada e molíbdica. Revista Ceres, v. 52, n. 01, p. 567-577, 2005. 